\title{
Carfilzomib combined with ex vivo-expanded patient autologous natural killer cells for myeloma immunotherapy
}

\author{
S. K. CHANG ${ }^{1, *}$, J. HOU ${ }^{1, *}$, G. G. CHEN ${ }^{1}$, D. D. YU ${ }^{1}$, H. Q. WU1, Y. S. XIE ${ }^{1}$, L. N. HU ${ }^{1}$, L. GAO ${ }^{1}$, W. Q. XIAO ${ }^{1}$, Y. Y. KONG ${ }^{1}$, Y. TAO ${ }^{1, *}$, J. M. SHI ${ }^{1,2, *}$ \\ ${ }^{1}$ Department of Hematology, Shanghai Tenth People's Hospital, Tongji University School of Medicine, Shanghai 200072, China; ${ }^{2}$ Tongji Univer- \\ sity Cancer Center, Tongji University, Shanghai 200092, China
}

*Correspondence: shijumei@tongji.edu.cn; taoyi018@139.com

"These authors contributed equally to this work.

Received October 19, 2017 / Accepted February 28, 2018

\begin{abstract}
Natural killer (NK) cell-based immunotherapy is promising, because NK cells form the first line of defense against cancer and are capable of lysing tumor cells without pre-stimulation. However, NK cells from multiple myeloma (MM) patients are always deficient in number, and the expression of certain activating receptors disables their cancer cyto-toxicity. Therefore, effective strategies to expand NK cells and increase NK cell-mediated cyto-toxicity against MM are imperative. Herein, NK cells were efficiently expanded from peripheral blood mononuclear cells (PBMCs) of newly diagnosed MM patients after co-culture with irradiated K562 cells transfected with 41BBL and membrane-bound interleukin (IL)-15 (K562-mb15-41BBL) in the presence of $200 \mathrm{IU} / \mathrm{ml}$ human IL-2. The ex vivo-expanded NK cells were demonstrated to vigorously kill both MM cells and autologous primary MM cells without significant lysis of normal patient cells. Further exploration revealed significant increase in cell surface expression of most activating receptors of NK cells and indicated that expanded NK (exp-NK) cell killing of MM cells was mediated by perforin/granzyme. NK cells are capable of lysing human leukocyte antigen (HLA) I-deficient tumor cells and carfizomib, a selective proteasome inhibitor approved for the treatment of relapsed/refractory MM patients down-regulates the expression of HLA class I, thus enhancing NK cell-mediated lysis in MM. Herein, we established for the first time that carfizomib dramatically augmented ex vivo exp-NK cell cytotoxicity against patient autologous MM cells, thus suggesting successful use of exp-NK alone or in combination with the drug in treating MM patient.
\end{abstract}

Key words: carfilzomib, natural killer cell, ex vivo expansion, multiple myeloma, immunotherapy, K562-mb15-41BBL

Multiple myeloma (MM) is a hematological malignancy of the B-cell line [1]. It is estimated that approximately 30,280 cases of myeloma are expected to be diagnosed in 2017 and almost 12,590 myeloma patients will die from the disease [2]. While autologous stem cell transplantation (ASCT) and high-dose chemotherapy have recently improved the overall survival of MM patients [3, 4], the minimal or measurable residual disease (MRD) followed by ASCT and the inevitable drug resistance to chemotherapeutic drugs are still major causes of MM progression and relapse $[5,6]$. Therefore, cancer immunotherapy capable of killing multi-drug-resistant malignant cells and stabilizing or even clearing the MRD should be an effective strategy for patients with relapsed/ refractory MM [7, 8].

Human natural killer $(\mathrm{NK})$ cells are $\mathrm{CD} 56^{+} \mathrm{CD} 3^{-}$large granular lymphocytes and an important subset of the innate immune system, capable of lysing certain tumor and virusinfected cells [9]. NK cells mainly kill target cells through perforin/granzymes granule-mediated exocytosis and death receptor interaction. Cytokines, such as Fas ligand, tumor necrosis factor (TNF) and the TNF-related apoptosisinducing ligand (TRAIL) expressed by NK cells interact with corresponding death receptors on target cells, resulting in NK cell-mediated killing of certain target cells [10]. The recognition of target cells by NK cells is regulated by a balance of positive and negative signals arising from various inhibitory and activating receptors on NK cells interacting with ligands on the target cells $[9,10]$.

Studies have reported that NK cells from the bone marrow of MM patients exert strong activity against myeloma cell lines [11]. Additionally, NK-cell cytotoxicity against MM positively correlates with the expression of activating recep- 
tors on the surface of NK cells and also with the number of cells $[12,13]$. However, the levels of NK cells in MM patients' peripheral blood show significant decrease [14] and NK cell activity is frequently impaired. This is significantly associated with advanced clinical stage [15]. Moreover, MM patients with lower NK cell activity have worse disease-free survival than those with higher NK cell activity [16]. Hence, effective strategies to expand NK cells and their cell-mediated cytotoxicity against MM are imperative.

Carfilzomib, a second-generation proteasome inhibitor (PI), has been approved to treat relapsed/refractory MM patients who have received at least two prior therapies, including the first-in-class PI bortezomib and an immunomodulatory drug [17]. We recently reported that carfizomib can enhance NK cell-mediated lysis of MM by decreasing HLA-class I expression, thus suggesting its use as a possible pharmaceutical-therapeutic approach to combine with NK cell-based therapies in MM [18].

Herein, irradiated K562 cells transfected with 41BBL and membrane-bound interleukin (IL)-15 (K562-mb15-41BBL) were used to expand NK cells from newly diagnosed MM patients' peripheral blood mononuclear cells (PBMCs). We then analyzed the cytotoxicity of the expanded NK cells against MM cells and the MM patient autologous cells and related mechanisms. Finally, the role of carfilzomib in enhancing cytotoxicity of ex vivo-expanded NK (exp-NK) cells against MM cells was investigated.

\section{Materials and methods}

Cells and cell culture. K562 and U266 cell lines were purchased from American Type Culture Collection (Manassas, VA). K562 transfectants (K562-mb15-41BBL), expressing membrane-bound interleukin (IL)-15 and the NK-stimulatory molecules 4-1BB ligand, were available in our laboratory. Peripheral blood and bone marrow specimens were obtained following acquisition of the study participants' written informed consent and the study was approved by the Shanghai Tenth People's Hospital Institutional Review Board. $\mathrm{CD}^{2} 6^{+} / \mathrm{CD}^{-} \mathrm{NK}$ cells and $\mathrm{CD} 138^{+} \mathrm{MM}$ cells were isolated from peripheral blood or bone marrow using magnetic bead selection (Miltenyi Biotech, Auburn, CA). All selected cells were routinely $>95 \%$ pure. All cell lines mentioned above were incubated with RPMi-1640 medium (Thermo Fisher Scientific, Waltham, MA, USA) supplemented with $10 \%$ heat-inactivated fetal bovine serum and $1 \%$ penicillin streptomycin-glutamine at $37^{\circ} \mathrm{C}$ with $5 \% \mathrm{CO}_{2}$.

Regents and antibodies. Carfizomib was purchased from Onyx Pharmaceuticals. Propidium iodide (PI), annexin $\mathrm{V}$ and fluorescence-conjugated-antibodies against CD3, CD14, CD19, CD33, CD56, CD138, NKG2D, DNAX accessory molecule-1 (DNAM-1), KIR2DL1 (CD158a), KIR2DL2/3(CD158b), KIR3DL1 (NKB1), perforin, granzyme A, granzyme B, TRAIL Fas-ligand (Fas-L) and isotype were purchased from BD Biosciences (San Jose,
CA). NKp30, NKp44, NKp46 antibodies were purchased from Beckman Coulter (Fullerton, CA). Concanamycin A (CMA, perforin/granzyme inhibitor) and HLA class I ABC monoclonal antibodies were purchased from Sigma (China Sigma Co., Ltd.).

Ex-vivo expansion of NK cells. The following culture conditions were used for ex vivo expansion of NK cell with leukapheresis product of PBMCs from newly diagnosed MM patients. Briefly, PBMCs $\left(1.5 \times 10^{6}\right.$ cells $\left./ \mathrm{ml}\right)$ were co-cultured with irradiated K562-mb15-41BBL (100 Gy, $1.0 \times 10^{6}$ cells $/ \mathrm{ml}$ ) at a ratio of 1.5:1 in RPMI 1640 medium (Thermo Fisher Scientific, Waltham, MA, USA) supplemented with $10 \%$ fetal bovine serum, $1 \%$ penicillin streptomycin (Gibco, Carlsbad, CA, USA) and $200 \mathrm{IU} / \mathrm{ml}$ recombinant human IL-2 (R\&D Systems, Minneapolis, MN, USA) at $37^{\circ} \mathrm{C}$ with $5 \% \mathrm{CO}_{2}$. Fifty percent of the medium was refreshed every second day. The cells were re-stimulated with K562-mb15-41BBL and $200 \mathrm{IU} / \mathrm{ml} \mathrm{IL}-2$ at day 7 and collected at day 14 for assays. Flow cytometry calculated the number of total viable cells and the proportion of each subset at day 0 and day 14 of co-cultures. The percentages of viable NK cells were determined by annexin-V/PI double-staining using Vybrant apoptosis assay kit (Invitrogen, Carlsbad, CA). Fold expansion was calculated as pre-expansion cells (day 0 of co-culture) divided by post-expansion cells (day 14 of co-culture).

Phenotyping of subset cells by flow cytometry analysis. Cell surface protein expression of NK cells was determined by standard flow cytometry analysis using isotype control or FITC, PE conjugated antibodies (Abs). In brief, pre-expansion (day 0 of co-culture) or post-expansion (day 14 of co-culture) cells were collected and re-suspended at a concentration of $1.0 \times 10^{6}$ cells $/ \mathrm{ml}$. The cells were then stained with $10 \mu \mathrm{l}$ fluorescence-conjugated CD3PerCp, CD56FITC antibody or $\mathrm{PE}$ conjugated antibodies against $\mathrm{NK}$ cell activating or inhibitory receptors at room temperature for 25 minutes, washed with phosphate buffer solution in triplicate and detected by flow cytometry (BD Biosciences, San Jose, CA, USA). For NK cell phenotyping, the culture cells at day 0 and day 14 were first T-cell depleted and then selected for NK cells by $\mathrm{CD}^{+} 6^{+}$selection using Miltenyi beads. The resulting cells comprised more than $95 \% \mathrm{CD}^{2} 6^{+} / \mathrm{CD}^{-} \mathrm{NK}$ cells. The mean fluorescence intensity (MFI) values were calculated for day 0 and day 14 samples for each cell surface receptor analyzed,. The changes in receptor expression was expressed as MFI ratios (MFI day 14/MFI day 0 ) for each receptor. MFI ratio $>1$ denoted up-regulation of the receptor while MFI ratio $<1$ indicated down-regulation in receptor expression

${ }^{51} \mathrm{Cr}$ release cytotoxicity assay. Cytotoxicity mediated by $\mathrm{CD}^{\circ} 6^{+} / \mathrm{CD}^{-} \mathrm{NK}$ cells against MM cell line U266, patient autologous MM cells and PBMCs was studied using a standard 4 -hour ${ }^{51} \mathrm{Cr}$ release assay. We used 10:1, 5:1 and 1:1 effector-to-target (E/T) ratios for all assays, except where otherwise indicated. Target cells were first labeled with $100 \mu \mathrm{Ci}{ }^{51} \mathrm{Cr}$ sodium chromate (Amersham Biosciences Europe $\mathrm{GmbH}$, Freiburg, Germany) for 1.5 hours and then 
washed with culture medium in triplicate. Then effector cells were co-cultured with target cells in a 96-well v-bottom plate. After 4 hours incubation, the supernatants were harvested for gamma count and the specific lysis percentage was calculated as (test release-spontaneous release)/(maximal releasespontaneous release) $\times 100$.

Molecular mechanisms involved in the interaction between exp-NK cells and patient MM cells were explored using blocking experiments. Exp-NK cells were first incubated with human IgG $\left(1 \mu \mathrm{g} / 10^{5}\right.$ cells, Invitrogen $)$ on ice for 20 minutes to avoid antibody-dependent cell-mediated cytotoxicity and then incubated with various $\mathrm{mAbs}$ at room temperature before addition of target cells. Isotype control or un-conjugated purified antibodies to NKG2D, natural cytotoxicity receptors (NKp30, NKp44 and NKp46) and DNAM-1 were used alone or in combination in NK cell receptor blocking experiments. The antagonistic TRAIL and Fas-L antibodies were used in the cytotoxic pathway blocking experiments. All mAbs were present at the final concentration of $10 \mu \mathrm{g} / \mathrm{ml}$.

Statistical analysis. Data obtained from multiple experiments were expressed as mean \pm standard deviation (SD). Significance levels were determined by Student $t$ test analysis with SPSS v22.0 (IBM, Armonk, NY, USA) and $\mathrm{p}<0.05$ was considered significant. All experiments were performed at least in triplicate.

\section{Results}

K562-mb15-41BBL cells efficiently expand NK cells from PBMCs of newly diagnosed MM patients. To explore the ability of K562-mb15-41BBL cells to specifically inducing proliferation of NK cell in vitro, PBMCs from six newly diagnosed MM patients were co-cultured with irradiated K562-mbIL15-41BBL cells at a ratio of 1.5: 1 in culture media containing $200 \mathrm{IU} / \mathrm{ml}$ human IL-2. After 14 day culture, we observed a mean of $270.3 \pm 84.3$ fold NK cell expansion (CD56 ${ }^{+} \mathrm{CD} 3-$ defined by flow cytometry), and NK cells were significantly enriched in cell products (day 14 with $86.1 \pm 5.3 \%$ versus day 0 with $11.6 \pm 3.1 \%$; $\mathrm{p}<0.05)$. No significant enrichment of NKT cells was observed $\left(\mathrm{CD} 56^{+} \mathrm{CD}^{+}-\right.$day 14 with $3.2 \pm 1.7 \%$ versus day 0 with $7.7 \% \pm 1.9 \%$ ). In contrast, the $\mathrm{CD} 3^{+} \mathrm{CD} 56^{-} \mathrm{T}$-cells had a mean expansion of only $4.1 \pm 2.5$ fold and the percentage of $\mathrm{T}$ cells in cell products reduced after 14 days of co-incubation (day 14 with $8.1 \pm 3.4 \%$ versus day 0 with $56.5 \pm 6.3 \%$ ).

At the end of co-culture, the cell populations contained only a mean of $2.1 \pm 1.2 \%$ B cells, $0.9 \pm 0.4 \%$ monocytes, and $3.1 \pm 5.7 \%$ myeloid cells. Table 1 shows the composition of cell population before and after expansion, with the cell populations' immunophenotypic features before and after expansion by co-culturing MM patient PBMCs with K562-mb15$41 \mathrm{BBL}$ cells in the presence of $200 \mathrm{IU} / \mathrm{ml}$ of IL-2 (one representative patient) presented in Figure 1A. Fujisaki et al. [19] support our results that cultures which had either non-transfected K562 with IL-2 or IL-2 alone failed to induce significant ( $\geq 2$-fold) NK cell proliferation (data not shown). The exp-NK cells were $>95 \%$ viable at harvest.

We also compared the immunophenotypic features of NK cells before and after expansion with K562-mb15-41BBL cells by flow cytometry. Figure $1 \mathrm{~B}$ histogram depicts the changes in cell surface expression of both activating and inhibitory receptors of NK cells of one representative MM patient. While the expression of activating receptors, including NKG2D, NKp30, NKp44 and DNAM-1, were significantly increased after 14 day co-culture, no obvious differences were defined in the expression of the NKp46 activating receptor or the inhibitory KIR2DL1 (CD158a), KIR2DL2 (CD158b) and KIR3DL1 (NKB1) receptors.

Exp-NK cells exert highly cytolytic activity against MM cell line U266 and patient autologous MM cells. Cytotoxicity assays were performed by ${ }^{51} \mathrm{Cr}$-release assay in order to investigate the anti-MM potential of exp-NK cells in vitro. Figure 2A shows the cytolytic capacity of exp-NK cells and non-exp-NK cells against MM cell line U266. Exp-NK cells had more vigorous cytotoxicity against U266 MM cell line compared to non-exp-NK cells. The mean lysis of U266 by exp-NK cells $(n=6)$ was $51.9 \pm 4.8 \%$ at a $10: 1$ E: T ratio,

Table 1. The composition of cell population before expansion and after expansion.

\begin{tabular}{|c|c|c|c|c|c|c|c|c|c|c|c|c|}
\hline \multirow[t]{2}{*}{ Donor } & \multicolumn{2}{|c|}{$\begin{array}{l}\mathrm{CD3}^{-} \mathrm{CD} 56^{+} \\
\text {NK cells }(\%)\end{array}$} & \multicolumn{2}{|c|}{$\begin{array}{c}\mathrm{CD3}^{+} \mathrm{CD}^{\circ} 6^{+} \\
\text {NKT cells }(\%)\end{array}$} & \multicolumn{2}{|c|}{$\begin{array}{c}\mathrm{CD3}^{+} \\
\text {T cells }(\%)\end{array}$} & \multicolumn{2}{|c|}{$\begin{array}{c}\mathrm{CD}^{\circ} 9^{+} \\
\text {B cells }(\%)\end{array}$} & \multicolumn{2}{|c|}{$\begin{array}{c}\mathrm{CD} 33^{+} \mathrm{CD} 14^{+} \\
\text {Monocytes (\%) }\end{array}$} & \multicolumn{2}{|c|}{$\begin{array}{c}\mathrm{CD}^{3} 3^{+} \mathrm{CD} 14^{-} \\
\text {Myeloid cells (\%) }\end{array}$} \\
\hline & Day 0 & Day 14 & Day 0 & Day 14 & Day 0 & Day 14 & Day 0 & Day 14 & Day 0 & Day 14 & Day 0 & Day 14 \\
\hline 1 & 10.3 & 81.6 & 6.2 & 3.4 & 46.2 & 12.4 & 27.1 & 1.7 & 9.3 & 0.8 & 1.2 & 0.7 \\
\hline 2 & 9.6 & 90.7 & 6.7 & 2.5 & 55.1 & 6.1 & 15 & 1.5 & 13.4 & 0.5 & 0.5 & 0.5 \\
\hline 3 & 11.4 & 78.5 & 4.6 & 2.3 & 62.8 & 11.3 & 10.3 & 4.1 & 8.6 & 1.6 & 2.5 & 1.1 \\
\hline 4 & 13.1 & 88.4 & 8.5 & 6.5 & 62 & 9.5 & 13.9 & 1 & 2.7 & 0.9 & 1.2 & 14.8 \\
\hline 5 & 16.9 & 92.2 & 9.5 & 2.4 & 53.2 & 3.7 & 8.4 & 1.5 & 10.8 & 0.7 & 2.1 & 1.2 \\
\hline 6 & 8.2 & 85.1 & 8.9 & 2.1 & 59.7 & 5.8 & 8.6 & 2.9 & 12.1 & 0.9 & 3.4 & 0.5 \\
\hline Mean & 11.6 & 86.1 & 7.4 & 3.2 & 56.5 & 8.1 & 13.9 & 2.1 & 9.5 & 0.9 & 1.8 & 3.1 \\
\hline SD & 3.1 & 5.3 & 1.9 & 1.7 & 6.3 & 3.4 & 7.0 & 1.2 & 3.8 & 0.4 & 1.1 & 5.7 \\
\hline
\end{tabular}

Abbreviations: NK, natural killer; NKT, natural killer T; SD, standard deviation. 


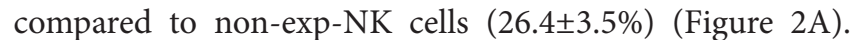
Similarly, 4-hour ${ }^{51} \mathrm{Cr}$ release assay also showed that autologous primary MM cells were avidly killed by exp-NK cells from MM patients $(n=4)$ at a 10:1 E: T ratio, whereas non-expNK cells exerted no cytotoxicity against these primary MM cells $(29.7 \pm 2.9 \%$ versus $1.7 \pm 0.7 \%, \mathrm{p}<0.05)$ (Figure $2 \mathrm{~B})$. We also tested the cytotoxicity of exp-NK cell against autologous PBMCs, and results indicated that exp-NK cells exerted no increased cytotoxicity against PBMCs compared to these non-exp-NK cells (data not shown). Our combined data indicates that exp-NK cells from MM patients stimulated with K562-mb15-41BBL cells can overcome the inhibitory signaling from autologous patient MM cells and have great potential in MM patient clinical immunotherapy.

Molecular mechanisms are involved in the interaction between exp-NK cells and patient MM cells. We next determined which NK activating receptors were involved in the enhanced lysis of primary autologous MM cells by exp-NK cells. Since activating receptor NKG2D, NKp30, NKp44, DNAM-1 were significantly up-regulated in exp-NK cells,

\section{A Before expansion}
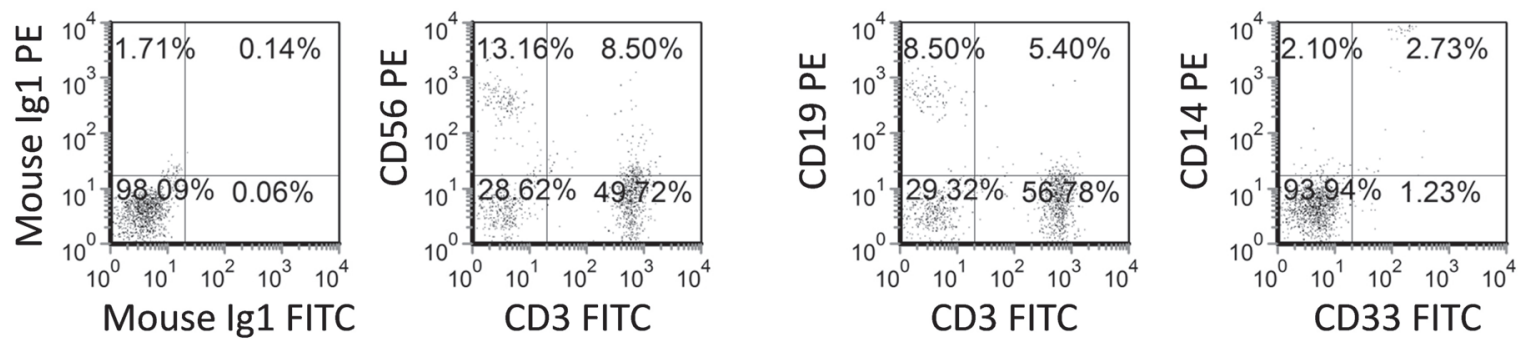

\section{After expansion}
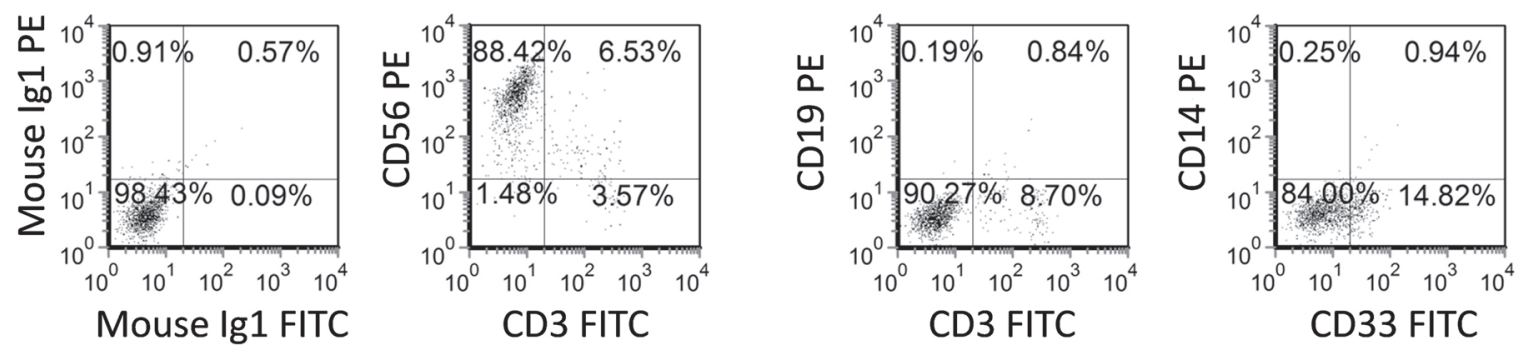

B
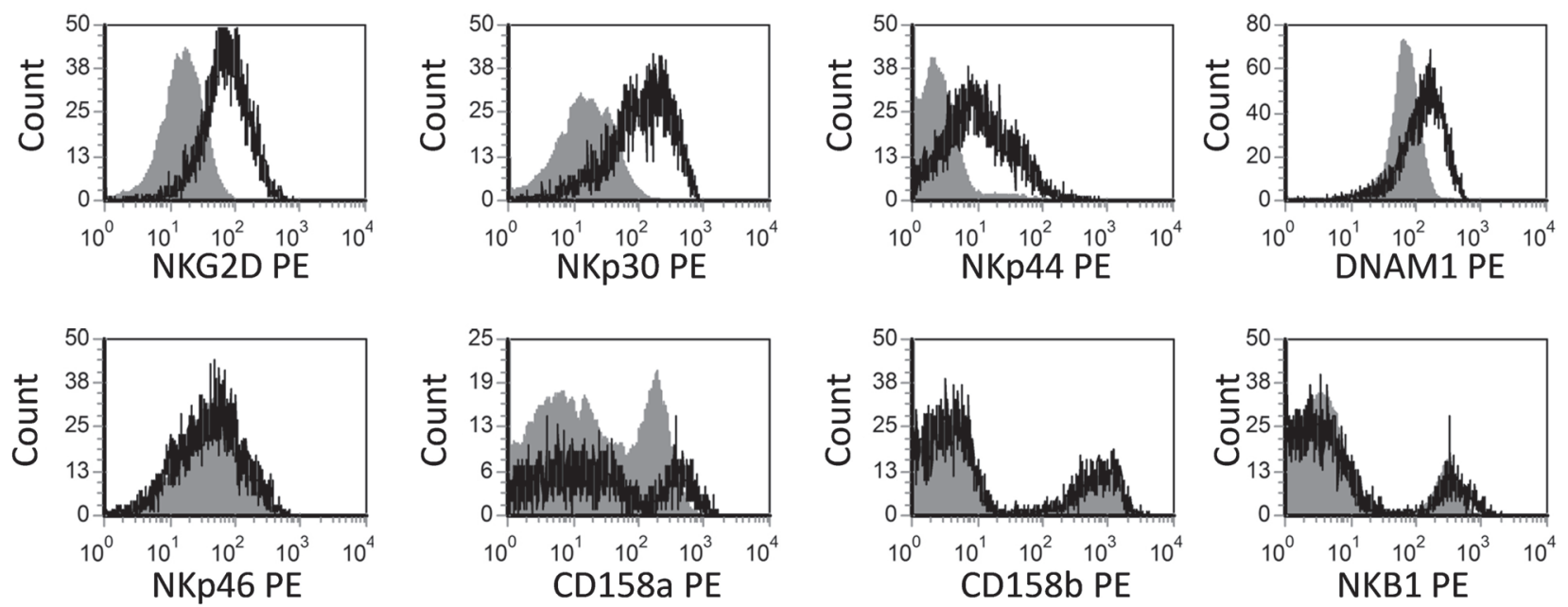

Figure 1. Phenotyping of subset cells before expansion and after expansion. PBMCs from newly diagnosed MM patients were co-cultured with irradiated K562-mbIL15-41BBL cells at a ratio of 1.5: 1 in culture media containing $200 \mathrm{IU} / \mathrm{ml}$ human IL-2. Cell surface protein expression was determined by standard flow cytometry analysis using isotype control or FITC, PE conjugated antibodies. A) Phenotyping of subset cells (CD56 ${ }^{+}$CD3- ${ }^{-}$NK cells, $\mathrm{CD} 6^{+} \mathrm{CD}^{+} \mathrm{NKT}$ cells, $\mathrm{CD}^{+} \mathrm{T}$ cells, $\mathrm{CD} 19^{+} \mathrm{B}$ cells, $\mathrm{CD}^{+} 3^{+} \mathrm{CD} 4^{+}$monocytes, and $\mathrm{CD}^{+} 3^{+} \mathrm{CD} 14^{-}$myeloid cells) from one representative patient before expansion and after expansion. B) Cell surface expression of activating and inhibitory receptors of exp-NK cells compared to non-exp NK cells of one representative MM patient. Open peaks show exp-NK cells and shaded peaks show non-exp NK cells. 

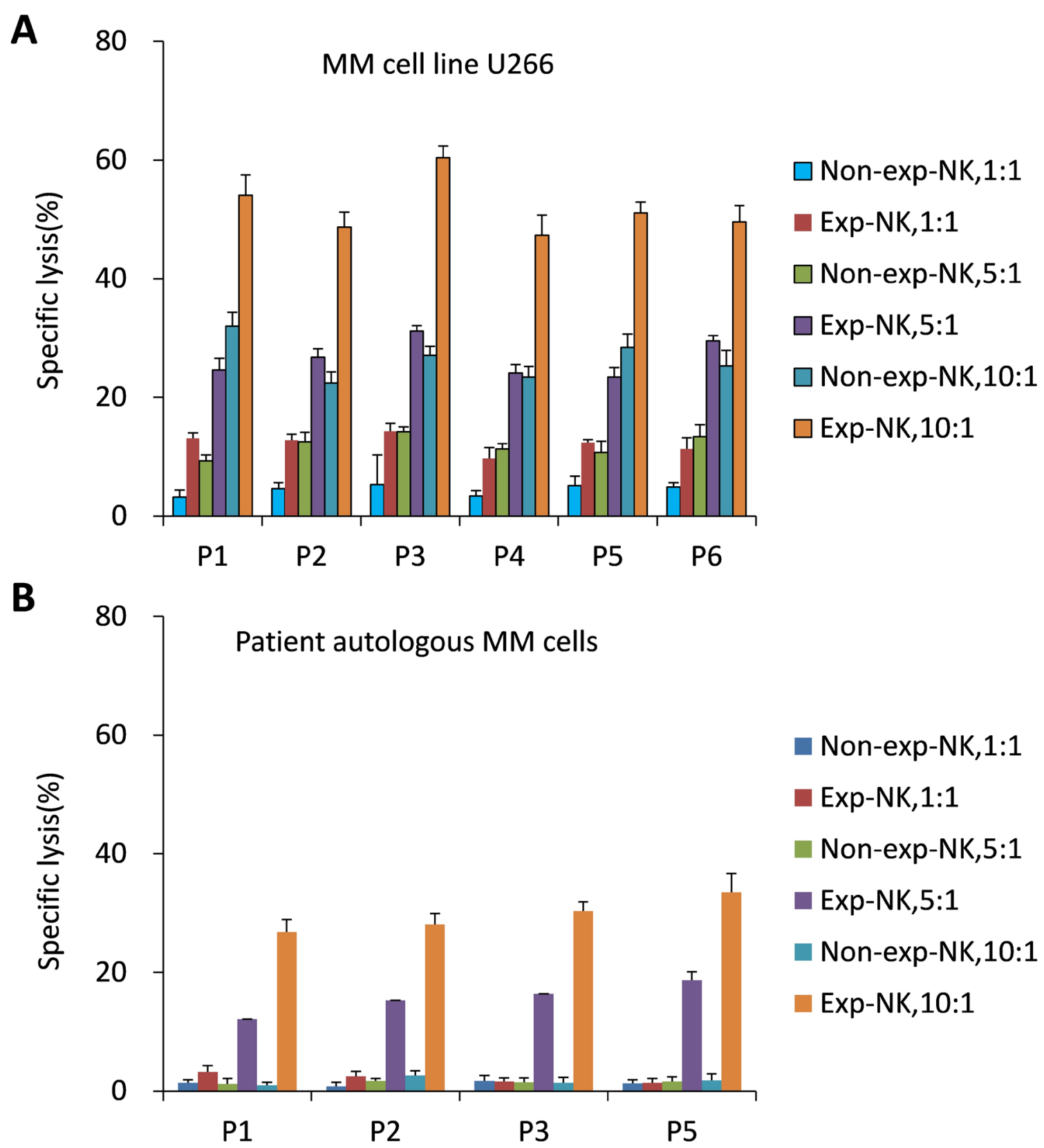

Figure 2. Exp-NK cells exerted highly cytolytic activity against MM cell line U266 and patient autologous MM cells. Cytolytic capacity of exp-NK cells against target cells was investigated using a standard 4-hour ${ }^{51} \mathrm{Cr}$ release assay at effector-to-target (E/T) ratios of 10:1, 5:1 and 1:1. A) Exp-NK cells from six MM patients showed more vigorous cytotoxicity against U266 MM cell line compared to non-exp-NK cells. B) The autologous primary MM cells were avidly killed by exp-NK cells from MM patients $(n=4)$ at a 10:1 E: T ratio, whereas non-exp-NK cells exerted no obvious cyto-toxicity against these primary MM cells.

we performed blocking studies with neutralizing antibodies, alone or in combination, to NKG2D, NKp30, NKp44, NKp46 and DNAM- 1 to evaluate the contribution of these activating receptors to the cytotoxicity of exp-NK cells against patient autologous MM cells.

As expected, exp-NK cell cytotoxicity was significantly reduced when activating receptor-ligand interactions were inhibited by these blocking antibodies. The killing rates of exp-NK cells in the presence of control antibody, anti-NKG2D antibody, anti-NKp30 antibody, anti-NKp44 antibody, anti-NKp46 antibody, anti-DNAM-1 antibody or all the antibodies together was $1.5 \pm 0.9 \%, 31.4 \pm 2.6 \%$, $21.3 \pm 1.5 \%, 15.4 \pm 1.9 \%, 2.2 \pm 0.5 \%, 34.1 \pm 2.6 \%$ and $75.7 \pm 3.8 \%$, respectively, at 10:1 E: T ratio (Figure $3 \mathrm{~A}$ ). 
Finally, blocking experiments were performed to identify the cytotoxic pathway involved in exp-NK cells. While the antagonistic TRAIL and Fas-L antibodies had no effect on exp-NK cell toxicity, the CMA perforin/granzyme inhibitor prevented enhancement of exp-NK cell-mediated lysis in patient MM cells (Figure 3B). This indicated that exp-NK cell killing of MM cells was mediated by perforin/granzyme. We also found that over $90 \%$ of exp-NK cells had high perforin, granzyme A and granzyme B expression and no notable expression of TRAIL and Fas-L was detected on exp-NK cells (Figure 3C).

Carfilzomib dramatically enhances ex vivo exp-NK cell cytotoxicity against patient autologous MM cells. We previously reported that MM cell treatment with carfizomib, a selective and irreversible proteasome inhibitor, downregulates HLA class I inhibitory killer cell immunoglobulinlike KIR-L on patient MM cells and this sensitizes patient $\mathrm{MM}$ cells to allogeneic NK cell cytotoxicity [18]. We therefore compared lysis by exp-NK cells vs. non-exp-NK cells against carfilzomib-treated and untreated autologous patient MM cells by testing HLA class I expression on patient MM cells treated with and without $10 \mathrm{nM}$ carfilzomib for 24 hours. We found HLA class I down-regulation after $10 \mathrm{nM}$ carfilzomib treatments in all carfilzomib treated myeloma cells $(n=4)$ with a mean of $55.8 \pm 10.2 \%$ (Figure $4 \mathrm{~A}$ ). We also observed that lysis of MM cells by both exp-NK cells and non-expNK cells was augmented by pre-treating tumor cells with 10 $\mathrm{nM}$ carfilzomib for 24 hours (Figure 4B). Interestingly, in contrast to non-exp-NK cells, there was a dramatic increase in carfilzomib-treated MM cell killing by exp-NK cells. At a 10:1 effector-to-target ratio, the mean lysis of exp-NK cells are $73.8 \pm 3.7 \%$ and $29.7 \pm 2.9 \%$ of carfilzomib-treated vs. untreated MM tumor cells respectively, compared to non-expNK cells which killed $24.9 \pm 6.0 \%$ and $1.7 \pm 0.7 \%(n=4, p<0.05)$ of carfilzomib-treated vs. untreated MM cells (Figure 4B).

\section{Discussion}

Increasing studies have recently focused on NK cellbased immunotherapy because NK cells are in the first line of defense against cancer and capable of lysing tumor cells without pre-stimulation [20], and previous preclinical and
A

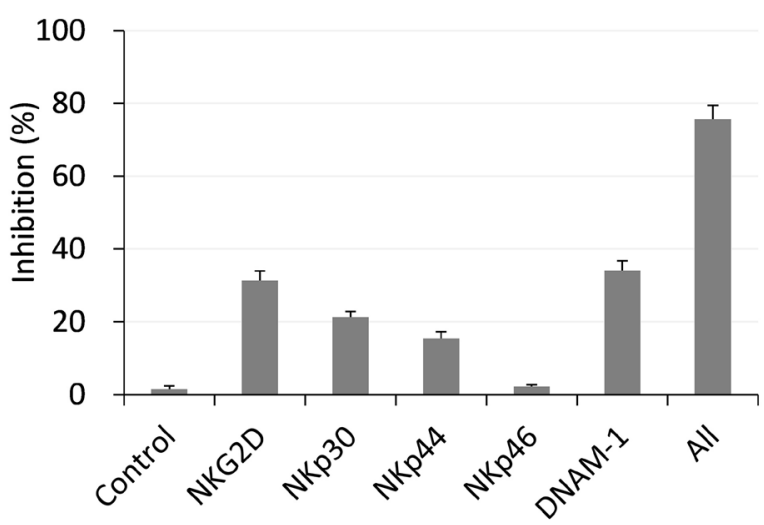

B

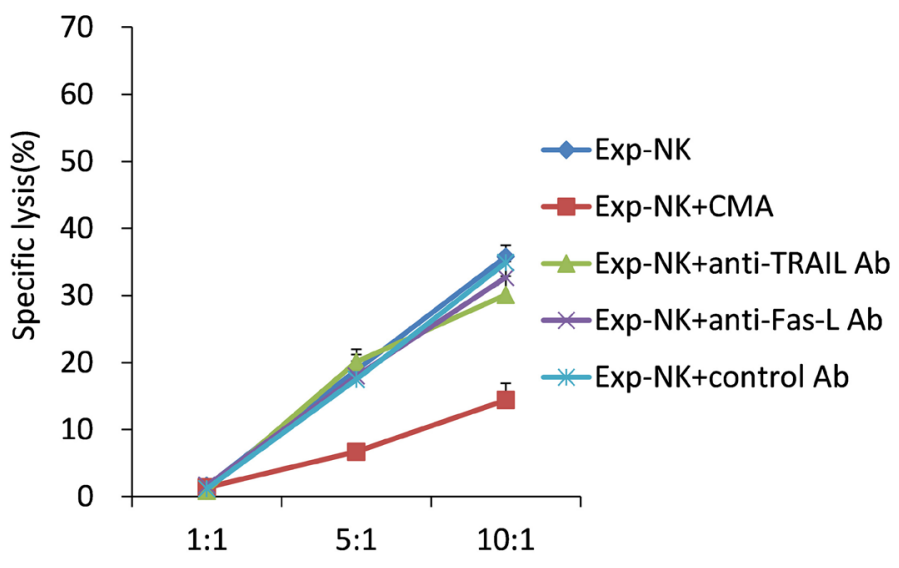

C
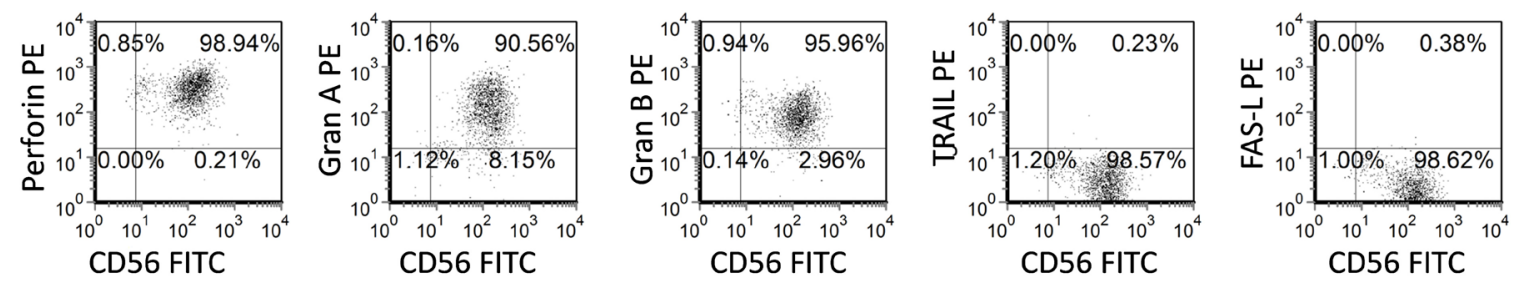

Figure 3. Molecular mechanisms involved in the interaction between exp-NK cells and patient MM cells. A) Blocking experiments by neutralizing antibodies NKG2D, NCRs (NKp30, NKp44 and NKp46) and DNAM-1 were performed alone or combined with isotype control to confirm the contribution of these activating receptors to the anti-MM activity of exp-NK cells. B) Blocking experiments by using antibodies to isotype control, TRAIL, Fas-L and the perforin/granzyme inhibitor CMA were performed to identify the cytotoxic pathway involved in exp-NK cells. C) Levels of expression of perforin, granzyme A, granzyme B, TRAIL and Fas-L were determined by flow cytometric analysis. 
A
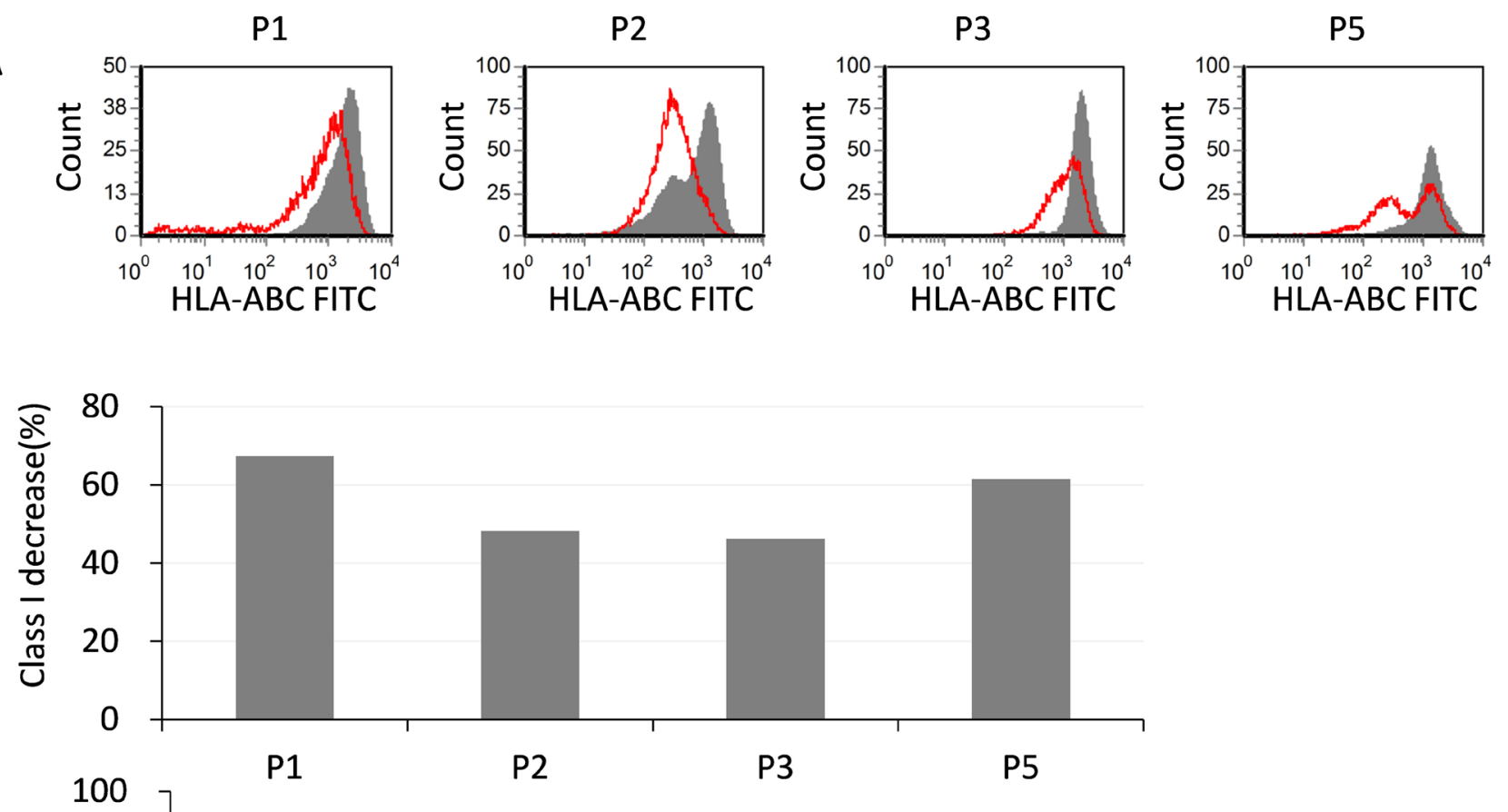

B

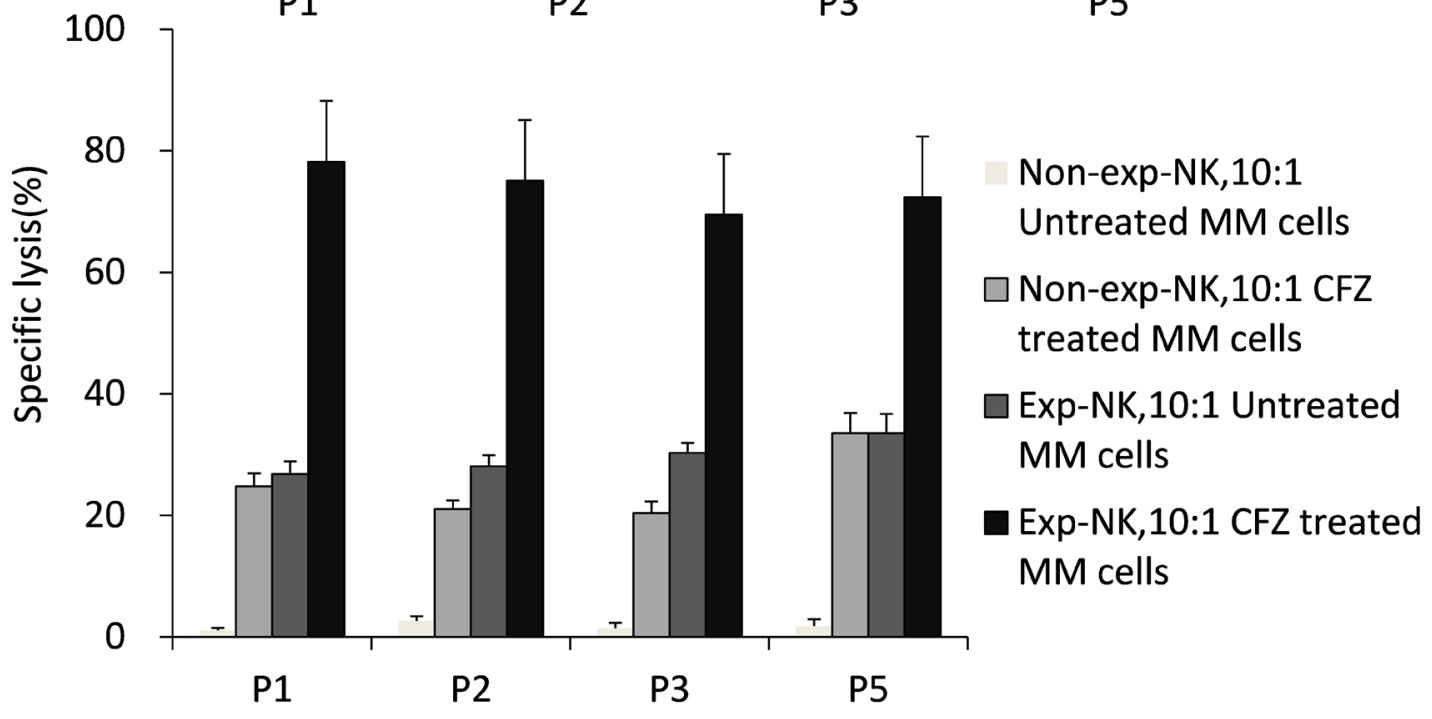

Figure 4. Carfilzomib dramatically enhanced ex vivo exp-NK cell cytotoxicity against patient autologous MM cells. A) Levels of expression of HLA class I on patient MM cells treated with and without $10 \mathrm{nM}$ carfilzomib were tested by flow cytometry. Open peaks show carfizomib-treated MM cells and shaded peaks are untreated cells. Histograms showed down-regulation of HLA class I ABC after carfilzomib treatment in all carfilzomib-treated MM cells $(n=4)$, by a mean of HLA class I down-regulation of $55.8 \pm 10.2 \%$. B) Primary MM cells were first treated with $10 \mathrm{nM}$ carfilzomib for 24 hours, and the cytolytic capability of exp-NK cells and non-exp-NK cells against treated or untreated cells was then investigated with ${ }^{51} \mathrm{Cr}$ release assay at the effector-to-target $(\mathrm{E} / \mathrm{T})$ ratios of 10:1.

clinical trials have proven that adoptive NK cells infusions are promising therapeutic strategies in the treatment of cancer patients $[21,22]$. While infusions of autologous NK cells into patients have been proven safe and tolerable [23] clinical application of the adoptive transfer of autologous NK cells is limited [24]. Large numbers of NK cells must be expanded by effective strategies to satisfy clinical requirements and clinical studies on adoptive autologous NK-cell infusions have demonstrated reduced NK cell anti-tumor effect [24]. The limited tumor suppression is mainly due to the inhibitory receptors on NK cells, killer cell immunoglobulin-like receptors (KIRs), which recognize HLA-class molecules on tumor cells and deliver a series of inhibitory signals preventing tumor cells from being lysed by autologous NK cells [25]. Therefore, all efforts must concentrate on enhancing NK cells anti-tumor effects. 
Previous studies have reported that irradiation of the genetically modified K562-mb15-41BBL cell line specifically induces proliferation of human NK cells and generates large numbers of ex vivo-expanded NK cells with enhanced cytotoxicity $[19,21]$. We previously found that human NK cells were significantly expanded by co-culturing PBMCs isolated from healthy donors with irradiated K562-mb1541BBL cells containing $200 \mathrm{IU} / \mathrm{ml}$ human IL-2. This was the first cytokine approved for use in patients and also one kind of interleukin able to expand NK cells [26]. Herein, PBMCs from newly diagnosed MM patients were co-cultured with irradiated K562-mbIL15-41BBL in the presence of IL-2, and we found significant expansion of NK cell from PBMCs of newly diagnosed MM patients. In contrast, significant enrichment of NKT, $\mathrm{T}$ and other cell types was not observed; thus highlighting the specific effects of this co-cultured irradiation system for NK cell expansion. Transfer of NK cells expanded from PBMCs of healthy donors (allogeneic NK cells) can exert enhanced tumor cytotoxicity by allogenic KIR ligand-incompatible NK cell [27]. However, the intense immuno-suppression required prior to infusions, and the risk of complicating life-threatening graft-versus-host disease, limit its clinical applications [20]. Therefore, ex vivo expansion of NK cell from the patient's own PBMCs (autologous NK cells) is not only conducive to increasing NK cells in numbers but it is also tolerated.

Evading NK cell-recognition and killing is essential for tumor formation and progression $[9,10]$. Previous studies have reported that NK cells from MM patients are always deficient in the expression of certain activating receptors and display weaker cytotoxicity against tumor cells, particularly in advanced stages $[14,15]$. Significant increase in the expression of most activating receptors including NKG2D, NKp30, NKp44 and DNAM-1 was observed by flow cytometry after 14 day co-culture, but no obvious change was seen in most inhibitory receptors. The immunoactivity of NK cells depends on a balance of activating and inhibitory signals [9], and once activating signals outbalance inhibitory signals, NK cells are activated. Our above results suggest that ex vivo exp-NK cells from MM patients may have great cytotoxicity against malignant cells. Therefore, ${ }^{51} \mathrm{Cr}$-release assay was performed to further evaluate the anti-MM potential of exp-NK cells in vitro and we found that exp-NK cells from MM patients avidly killed patient MM cells better than non-expanded NK cells without significant lysis of autologous PBMCs. Moreover, our preliminary data highlighted that expanded NK cells from MM patient have markedly cytolytic function against autologous primary MM cells; thus suggesting this treatment could be extended to most individual patients and the requirement for an available appropriate NK cell donor avoided.

The C-type lectin-like NKG2D, DNAX accessory molecule-1 (DNAM-1) and natural cytotoxicity receptors (NCRs) NKp30, NKp44, and NKp46 are common activating receptors expressed on NK cells and have important roles in the full activation of NK cells $[9,10,27]$. As mentioned above, a significant increase in the expression of most activating receptors was observed in our study, so we further explored the molecular mechanisms involved in the interaction between exp-NK cells and patient MM cells by blocking studies with specific antibodies against these activating receptors. Approximately $75 \%$ abrogation of enhanced killing of MM was determined in the presence of all Abs tested, and this suggests synergy between activating receptors responsible for marked increases in anti-MM activity in exp-NK cells. Other activated molecules may also be involved in the killing effects of exp-NK. Activated NK cells can release cytotoxic granules containing perforin and granzymes or generate death receptor ligands such as Fas-L and TRAIL, causing direct lysis of malignant cells [10]. Herein, we found that perforin/granzyme participated in exp-NK cell anti-MM activity, but Fas-L and TRAIL did not.

The cytolytic capacity of autologous NK cells is limited because of the inhibitory receptors KIRs interaction with self-HLA molecule effect on tumor cells [25]. It has been demonstrated that NK cells are capable of lysing HLA I-deficient tumor cells [28], and we recently established that carfizomib, a selective proteasome inhibitor approved for treatment of relapsed/refractory MM patients, downregulates MM expression of HLA class I and enhances NK cell-mediated lysis [18]. This finding suggests that the combination of carfizomib with autologous NK cells enhances effective MM therapy. Herein, we found for the first time that carfizomib dramatically augmented ex vivo exp-NK cell cyto-toxicity against patient autologous MM cells.

Previous studies have reported that infusions of NK cells combined with antitumor drugs produce no relevant toxic effects $[21,29]$. Therefore, the combination of carfizomib with ex vivo-expanded patient autologous NK cells has potential therapeutic implications for MM in the context of autologous adoptively transferred NK cells.

In conclusion, stimulation with K562 transfectants induces vigorous specific expansion of NK cells from MM patients without expanding $\mathrm{T}$ lymphocytes. The exp-NK cells exert highly cytolytic activity against both MM cells and autologous primary MM cells while sparing patient normal cells. Additionally, the exp-NK cell-killing of MM cells is mediated by perforin/granzyme. Significantly, carfilzomib dramatically enhances ex vivo exp-NK cell cytotoxicity against patient autologous MM cells. We believe that expanded/activated NK cells have a role in future treatment of MM patients with high-risk disease or other NK cellsensitive malignancies, either alone or in combination with carfizomib.

Acknowledgements: This work was supported by grants from the National Natural Science Foundation of China (81529001, 81670194 and 81570190). 


\section{References}

[1] BLADE J, CIBEIRA MT, FERNANDEZ DE LARREA C, ROSINOL L. Multiple myeloma. Ann Oncol 2010; 21: 313-319. https://doi.org/10.1093/annonc/mdq363

[2] SIEGEL RL, MILLER KD, JEMAL A. Cancer Statistics, 2017. CA Cancer J Clin 2017; 67: 7-30. https://doi.org/10.3322/ caac. 21387

[3] MERZ M, NEBEN K, RAAB MS, SAUER S, EGERER G et al. Autologous stem cell transplantation for elderly patients with newly diagnosed multiple myeloma in the era of novel agents. Ann Oncol 2014; 25: 189-195. https://doi. org/10.1093/annonc/mdt509

[4] CHILD JA, MORGAN GJ, DAVIES FE, OWEN RG, BELL SE et al. High-dose chemotherapy with hematopoietic stemcell rescue for multiple myeloma. N Engl J Med 2003; 348: 1875-1883. https://doi.org/10.1056/NEJMoa022340

[5] HART AJ, JAGASIA MH, KIM AS, MOSSE CA, SAVANI BN et al. Minimal residual disease in myeloma: are we there yet? Biol Blood Marrow Transplant 2012; 18: 1790-1799. https:// doi.org/10.1016/j.bbmt.2012.05.009

[6] KUSENDA J, KOVARIKOVA A. Multiple myeloma, immunotherapy and minimal residual disease. Neoplasma 2016; 63: 651-658. https://doi.org/10.4149/neo_2016_501

[7] TREICHEL RS, OLKEN S. The relationship between multidrug resistance and resistance to natural-killer-cell and lymphokine-activated killer-cell lysis in human leukemic cell lines. Int J Cancer 1992; 50: 305-310.

[8] SONG W, VAN DER VLIET HJ, TAI YT, PRABHALA R, WANG R et al. Generation of antitumor invariant natural killer $\mathrm{T}$ cell lines in multiple myeloma and promotion of their functions via lenalidomide: a strategy for immunotherapy. Clin Cancer Res 2008; 14: 6955-6962. https://doi. org/10.1158/1078-0432.CCR-07-5290

[9] HALLETT WH, MURPHY WJ. Positive and negative regulation of Natural Killer cells: therapeutic implications. Semin Cancer Biol 2006; 16: 367-382. https://doi.org/10.1016/j. semcancer.2006.07.003

[10] SMYTH MJ, CRETNEY E, KELLY JM, WESTWOOD JA, STREET SE et al. Activation of NK cell cytotoxicity. Mol Immunol 2005; 42: 501-510. https://doi.org/10.1016/j.molimm.2004.07.034

[11] UCHIDA A, YAGITA M, SUGIYAMA H, HOSHINO T, MOORE M. Strong natural killer (NK) cell activity in bone marrow of myeloma patients: accelerated maturation of bone marrow NK cells and their interaction with other bone marrow cells. Int J Cancer 1984; 34: 375-381.

[12] BERNAL M, GARRIDO P, JIMENEZ P, CARRETERO R, ALMAGRO $\mathrm{M}$ et al. Changes in activatory and inhibitory natural killer (NK) receptors may induce progression to multiple myeloma: implications for tumor evasion of $\mathrm{T}$ and NK cells. Hum Immunol 2009; 70: 854-857. https://doi. org/10.1016/j.humimm.2009.07.004

[13] EL-SHERBINY YM, MEADE JL, HOLMES TD, MCGONAGLE D, MACKIE SL et al. The requirement for DNAM-1, NKG2D, and NKp46 in the natural killer cell-mediated killing of myeloma cells. Cancer Res 2007; 67: 8444-8449. https://doi.org/10.1158/0008-5472.CAN-06-4230
[14] FAURIAT C, MALLET F, OLIVE D, COSTELLO RT. Impaired activating receptor expression pattern in natural killer cells from patients with multiple myeloma. Leukemia 2006; 20: 732-733. https://doi.org/10.1038/sj.leu.2404096

[15] JURISIC V, SRDIC T, KONJEVIC G, MARKOVIC O, COLOVIC M. Clinical stage-depending decrease of NK cell activity in multiple myeloma patients. Med Oncol 2007; 24: 312-317.

[16] PESSOA DE MAGALHAES RJ, VIDRIALES MB, PAIVA B, FERNANDEZ-GIMENEZ C, GARCIA-SANZ R et al. Analysis of the immune system of multiple myeloma patients achieving long-term disease control by multidimensional flow cytometry. Haematologica 2013; 98: 79-86. https://doi. org/10.3324/haematol.2012.067272

[17] LAROCCA A, MINA R, GAY F, BRINGHEN S, BOCCADORO M. Emerging drugs and combinations to treat multiple myeloma. Oncotarget 2017; 8: 60656-60672. https://doi. org/10.18632/oncotarget.19269

[18] YANG G, GAO M, ZHANG Y, KONG Y, GAO L et al. Carfilzomib enhances natural killer cell-mediated lysis of myeloma linked with decreasing expression of HLA class I. Oncotarget 2015; 6: 26982-26994. https://doi.org/10.18632/ oncotarget.4831

[19] FUJISAKI H, KAKUDA H, SHIMASAKI N, IMAI C, MA $J$ et al. Expansion of highly cytotoxic human natural killer cells for cancer cell therapy. Cancer Res 2009; 69: 4010-4017. https://doi.org/10.1158/0008-5472.CAN-08-3712

[20] FANG F, XIAO W, TIAN Z. NK cell-based immunotherapy for cancer. Semin Immunol 2017; 31: 37-54. https://doi. org/10.1016/j.smim.2017.07.009

[21] LEIVAS A, PEREZ-MARTINEZ A, BLANCHARD MJ, MARTIN-CLAVERO E, FERNANDEZ L et al. Novel treatment strategy with autologous activated and expanded natural killer cells plus anti-myeloma drugs for multiple myeloma. Oncoimmunology 2016; 5: e1250051. https://doi.org/10 $.1080 / 2162402 X .2016 .1250051$

[22] ALICI E, KONSTANTINIDIS KV, SUTLU T, AINTS A, GAHRTON G et al. Anti-myeloma activity of endogenous and adoptively transferred activated natural killer cells in experimental multiple myeloma model. Exp Hematol 2007; 35: 1839-1846. https://doi.org/10.1016/j.exphem.2007.08.006

[23] LUNDQVIST A, BERG M, SMITH A, CHILDS RW. Bortezomib Treatment to Potentiate the Anti-tumor Immunity of Ex-vivo Expanded Adoptively Infused Autologous Natural Killer Cells. J Cancer 2011; 2: 383-385.

[24] GRANZIN M, WAGNER J, KOHL U, CERWENKA A, HUPPERT V et al. Shaping of Natural Killer Cell Antitumor Activity by Ex Vivo Cultivation. Front Immunol 2017; 8: 458. https://doi.org/10.3389/fimmu.2017.00458

[25] BAKKER AB, PHILLIPS JH, FIGDOR CG, LANIER LL. Killer cell inhibitory receptors for MHC class I molecules regulate lysis of melanoma cells mediated by NK cells, gamma delta T cells, and antigen-specific CTL. J Immunol 1998; 160: 5239-5245.

[26] CHEN GG, WEI R, GAO L, HOU J, WU HQ et al. Enhanced cytotoxicity and underlying mechanism of ex vivo expanded natural killer cells against breast cancer cells. Int J Clin Exp Pathol 2017; 10: 6589-6600. 
[27] MILLER IS，SOIGNIER Y，PANOSKALTSIS-MORTARI A, MCNEARNEY SA, YUN GH et al. Successful adoptive transfer and in vivo expansion of human haploidentical NK cells in patients with cancer. Blood 2005; 105: 3051-3057. https://doi.org/10.1182/blood-2004-07-2974

[28] BENJAMIN JE, GILL S, NEGRIN RS. Biology and clinical effects of natural killer cells in allogeneic transplantation. Curr Opin Oncol 2010; 22: 130-137. https://doi.org/10.1097/ CCO.0b013e328335a559
[29] SZMANIA S, LAPTEVA N, GARG T, GREENWAY A, LINGO J et al. Ex vivo-expanded natural killer cells demonstrate robust proliferation in vivo in high-risk relapsed multiple myeloma patients. J Immunother 2015; 38: 24-36. https:// doi.org/10.1097/CJI.0000000000000059 